\title{
A resolução de problemas como estratégia metodológica de ensino, fundamentada na teoria da aprendizagem significativa: uma análise quantitativa
}

Jeneffer Araújo de Assunção jenefferassuncao@gmail.com 0000-0003-2144-9408

Universidade Federal de Roraima, Boa Vista, Roraima, Brasil.

Marco Antonio Moreira moreira@if.ufrgs.br

0000-0003-2989-619x

Universidade Federal do Rio Grande do

Sul, Porto Alegre, Rio Grande do Sul,

Brasil.

Concesa Caballero Sahelices concesa@ubu.es

0000-0001-8079-4717

Universidade de Burgos, Burgos, Burgos, Espanha.

\section{RESUMO}

No campo científico, atualmente existe a discussão importante sobre o ensino de funções através da metodologia da resolução de problemas. Este estudo tem como objetivo analisar a eficiência da estratégia de resolução de problemas como metodologia de ensino, fundamentada na teoria da aprendizagem significativa de Ausubel. Os sujeitos da pesquisa foram 84 estudantes da 1 o série do Ensino Médio de um Colégio Militar da PMRR, distribuídos em quatro turmas, que constituíram o grupo experimental e grupo controle, nas quais se desenvolveram os conteúdos de função e função afim. Os resultados foram analisados com base na estatística descritiva e ANCOVA com o intuito de avaliar a efetividade da metodologia de resolução de problemas fundamentada na teoria de aprendizagem significativa vis-à-vis a metodologia tradicional. Constatou-se que a aprendizagem dos conteúdos do grupo experimental, após a intervenção, pareceu apresentar desempenho superior nos testes do que o grupo controle.
\end{abstract}

PALAVRAS-CHAVE: Aprendizagem Significativa. Resolução de Problemas. Conceito de Função. 


\section{INTRODUÇÃO}

O processo de ensino e aprendizagem deve estar fundamentado por teorias de aprendizagem, pois a estrutura cognitiva é um complexo organizado de ideias, logo, é importante saber como o estudante armazena as informações em sua estrutura cognitiva e de que forma o professor deve conduzir este processo.

A Psicologia Cognitiva estuda como as pessoas percebem, aprendem, lembram e pensam sobre a informação. Tenta entender como e por que do pensamento, ou seja, os pesquisadores buscam formas de explicar e descrever a cognição (STERNBERG, 2010). A Psicologia cognitiva estuda o processo de aprendizagem, enfatizando de várias maneiras o importante papel que tem as ações vinculadas com a resolução de problemas para desenvolver aprendizagem mais efetiva. Diversos estudiosos sugerem ao professor a utilização das teorias de aprendizagem, pois essas permitem explicar processos mentais na aquisição do conhecimento.

A teoria da aprendizagem significativa tem destaque especial, a qual explica com detalhes, como se dá o processo de interação entre os conhecimentos a serem aprendidos e os já estabelecidos, uma vez que dá subsídios para o professor avaliar a aprendizagem significativa dos estudantes, diferenciando-a da aprendizagem mecânica.

Uma estratégia metodológica interessante é a resolução de problema como um caso especial de aprendizagem significativa, diante disto, surge a problemática da pesquisa: A implementação de uma estratégia de resolução de problemas como metodologia de ensino, fundamentada na teoria da aprendizagem significativa de Ausubel, possibilitará aos estudantes da 1a série do Ensino Médio aprender significativamente o conteúdo de função?

Este estudo é resultado de pesquisas realizadas durante o doutorado em Educação da Universidade de Burgos, no qual, serão explanadas definições essenciais da teoria da aprendizagem significativa, em seguida, a resolução de problemas como metodologia de ensino fundamentada na teoria da aprendizagem significativa.

\section{A RESOLUÇÃO DE PROBLEMAS COMO ESTRATÉGIA METODOLÓGICA DE ENSINO FUNDAMENTADA NA TEORIA DA APRENDIZAGEM SIGNIFICATIVA}

Para Ausubel et al. (1980), a aprendizagem significativa é um processo que permite relacionar um novo conhecimento, de maneira não-arbitrária e substantiva, a um aspecto relevante da estrutura cognitiva do indivíduo (MOREIRA, 2006, p. 08). Não-arbitrária nos transmite ideias de interações não aleatória sem uma concordância entre os conhecimentos, ou seja, não é com qualquer conhecimento prévio que o novo conhecimento vai interagir, e sim com o mais relevante disponível na estrutura cognitiva, no qual servirá de ponte de ancoradouro com os novos conhecimentos a serem apresentados ao estudante.

Neste aspecto, enfatiza-se a importância de averiguar o conhecimento prévio do estudante para que possa haver uma interação entre o que o estudante tem de conhecimento específico mais relevante, disposto na sua estrutura cognitiva e o novo conhecimento a ser-lhe apresentado, a qual Ausubel chama de subsunçor existente na estrutura cognitiva, estes subsunçores são os conhecimentos prévios 
especificamente relevantes. "Essa interação entre o novo conhecimento e o conhecimento prévio é que permite ao estudante atribuir significado ao novo conhecimento" (MOREIRA, 2006, p. 09).

Logo, a resolução de problemas é uma forma na qual o professor pode averiguar os conhecimentos prévios dos estudantes a partir de situações problema. Ausubel et al. (1980), descreve que a resolução de problemas representa uma forma de atividade ou pensamento dirigido, na qual tanto a representação cognitiva da experiência prévia como os componentes da situaçãoproblema são reorganizados, transformados ou recombinados para assegurar um determinado objetivo.

Sendo assim, a estrutura cognitiva preexistente desempenha papel preponderante na resolução de problemas, ainda mais se levado em conta que a busca de solução de qualquer problema envolve uma readaptação do resíduo da experiência prévia frente às demandas da nova situação-problema. Resolver um problema pode ser encarado como um meio para promover tal aprendizagem. Por exemplo, o surgimento do insight, conforme a concepção de Ausubel (2003), resulta de um processo de clarificação progressiva sobre relações de meio-e-fim fundamentadas na formulação, verificação e rejeição de hipóteses alternativas. Esta tarefa requer incorporação da nova informação na estrutura cognitiva do sujeito que a realiza.

Não se pode deixar de salientar a importância da linguagem na resolução de problemas, pois esta desempenha um papel importante na verbalização de conceitos ou proposições que resultam das operações de transformação envolvidas no pensamento. Ausubel et al. (1980), aponta que os tipos mais simples de raciocínio dependem apenas de operações relativamente concretas, perceptuais e imaginativas e, podem ser mais evidentes na ação antes da emergência do pensamento verbal, enquanto que a capacidade para pensar em termos abstratos obviamente requer o uso de conceitos e símbolos abstratos. "Somente os tipos mais primitivos de solução de problemas são possíveis sem a linguagem" (Ausubel et al. 1980, p. 412).

De acordo com Dante (2008), a resolução de problemas como metodologia de ensino auxilia o estudante na apreensão de significados, estimulando-o no desenvolvimento do raciocínio lógico, a saber enfrentar novas situações, preparando o cidadão para vida. Para Costa (2008, p. 264), a resolução de problemas em sala de aula é uma habilidade pela qual o indivíduo externaliza o processo construtivo de aprender, de converter em ações, conceitos, proposições e exemplos adquiridos através da interação com professores, pares e materiais instrucionais.

A resolução de problemas refere-se a "qualquer atividade na qual tanto a representação cognitiva de experiência prévia e os componentes de uma situação problema apresentada são reorganizados a fim de atingir um determinado objetivo" (AUSUBEL, 1980 apud COSTA, 2008, p. 194).

A resolução de problemas como estratégia metodológica de ensino contribui para a aprendizagem significativa, onde a busca da solução de qualquer problema envolve uma readaptação do resíduo da experiência prévia frente às novas situações a serem enfrentadas, na medida em que propicia reorganizar a informação ou o conhecimento armazenado na estrutura cognitiva do estudante. 
Costa (2008, p. 264) reitera que, "o surgimento do "insight", conforme a concepção de Ausubel, resulta de um processo de clarificação progressiva sobre relações de meio-e-fim fundamentada na formulação, verificação e rejeição de hipóteses alternativas".

Zompero e Laburu (2010, p. 34), "consideram que a resolução de problemas além de proporcionar o desenvolvimento do pensamento crítico dos estudantes, possibilita-os aplicar seus conhecimentos em novas situações". Sendo assim, essa metodologia pode ser utilizada pelo professor para verificar a aprendizagem de seus alunos, por requerer dos mesmos a aplicação dos conhecimentos em situações não familiares a eles, sendo esse um dos pressupostos da teoria da aprendizagem significativa.

Um dos autores pioneiros na pesquisa nessa área é o matemático George Polya. Em sua obra mais famosa How to Solve It, traduzida para o português como A arte de resolver problemas (POLYA, 1995), Polya se propõe a estudar os inúmeros métodos de resolução de problemas, estudo também conhecido como heurística, e suas implicações para o ensino e aprendizagem de Matemática. Com o objetivo de sistematizar o complexo processo que envolve a resolução de um problema matemático, o autor propõe um esquema no qual o mesmo pode ser resumido em quatro etapas: 1) Compreensão do problema, 2) Estabelecimento de um plano, 3) Execução do plano e 4) Retrospecto.

Contudo, para Mendoza (2009, p.70), "a resolução de problemas conforme foi proposta por Polya, não utiliza a formação das atividades de um determinado conteúdo com os respectivos elementos que caracterizam a ação". E ainda Talizina (1988), critica os trabalhos de Polya. Para a autora, estes trabalhos supõem tacitamente que os "alunos são capazes de realizar a atividade indispensável, se considerar o pensamento como certa função abstrata já existente e que a tarefa consiste só em fazê-lo trabalhar na direção necessária" (TALIZINA, 1988, p. 202).

Mendoza (2009), a partir de Polya, adaptou dos princípios gerais para resolver problemas, criando um esboço dos passos que conduz à solução de problemas fornecendo possibilidades que poderão ser úteis na resolução. Em sua observância Mendoza destacou que "os princípios sequenciais de Polya ao passar de um estágio para o outro, que caracteriza cada estágio como ação, envolve um conjunto de operações que estão diretamente ligadas com a realização desta ação" (MENDOZA, 2009, p.77).

A partir dos pressupostos existentes no método dos princípios de Polya, Mendoza construiu a Atividade de Situações Problema (ASP) formada por um sistema de ações, que foi utilizado na intervenção desta pesquisa, para desenvolver a capacidade dos estudantes de resolver problemas no conteúdo de função e função afim, utilizando como direcionamento a teoria da aprendizagem significativa, promovendo também a formação das ideias conceituais de função.

Em cada um dos procedimentos sequenciais, existe um conjunto de perguntas e indagações (operações), que direciona o estudante a encontrar a solução do problema. Estes princípios na perspectiva de Mendoza (2009), foram descritos para melhor entendimento, no Quadro 1, que explicita as ações e operações da atividade de situações problemas. 
Quadro 1 - Atividade de Situações Problema

Ações

1a Ação: Compreender
o problema

\begin{tabular}{c}
\hline 2a Ação: \\
\hline Construir o modelo \\
matemático
\end{tabular}

Ler o problema e extrair todos os elementos desconhecidos; estudar os dados e suas condições e determinar o(s) objetivo(s) do problema.

Determinar as variáveis e incógnitas; nominar as variáveis e incógnitas com suas unidades de medidas; construir o modelo matemático a partir das variáveis, incógnitas e condições e por último realizar a análise das unidades de medidas do modelo matemático.

\begin{tabular}{|c|c|}
\hline $\begin{array}{c}\text { 3a Ação: } \\
\begin{array}{c}\text { Solucionar o modelo } \\
\text { matemático }\end{array}\end{array}$ & $\begin{array}{c}\text { Selecionar o(s) método(s) matemático(s) para solucionar o } \\
\text { modelo. }\end{array}$ \\
\hline $\begin{array}{c}\text { Interpaço: } \\
\text { Interpretar o resultado; extrair os resultados significativos } \\
\text { que tenham relação com o(s) objetivo(s) do problema; dar } \\
\text { resposta ao(s) objetivo(s) do problema; realizar uma reflexão } \\
\text { baseado no(s) objetivo(s) do problema; analisar a partir de } \\
\text { novos dados e condições que tenham relação direta ou não } \\
\text { com o(s) objetivo(s) do problema existindo a possibilidade de } \\
\text { reformular o problema e assim construir novamente o } \\
\text { modelo matemático, solucioná-lo e interpretar sua solução. }\end{array}$ \\
\hline
\end{tabular}

Fonte: Mendoza (2009).

Primeira ação: compreender o problema. As operações propostas nesta ação são formadas com o intuito de assegurar que o estudante busque elementos que facilitem a compreensão do problema, destacando inicialmente uma leitura que permita extrair elementos conhecidos e desconhecidos; destacar para estudar e compreender os elementos desconhecidos; determinar os dados essenciais e as condições; e para finalizar a ação determinar o(s) objetivo(s) do problema. "Através desta análise, destaca-se também a observação quanto à notação matemática apropriada ou a realização de um diagrama se for o caso' (MENDOZA, 2009, p. 79).

Segunda Ação: construir o modelo matemático. Neste estágio acontece a relação ou associação dos elementos dados no problema, com o objetivo para solucionar, por isso, torna-se importante determinar as variáveis e incógnitas certificando-se das medidas e itens relacionados; nomear as variáveis e incógnitas com suas unidades de medida, atribuindo símbolos frequentemente utilizados, tais como $a, x, y, z$ ou atribuir iniciais como símbolos sugestivos volume (v), tempo (t), massa $(m)$, nesta ação, Mendoza enfatiza que:

\footnotetext{
Construir o modelo matemático a partir das variáveis, incógnitas e condições do problema. É uma operação entendida como fator essencial desta ação, pois requer do estudante o reconhecimento de padrões que ocorrem no contexto do problema, que pode ser geométrico, numérico ou algébrico, após a identificação do padrão, o estudante pode determinar o modelo matemático ou conjecturar se for o caso. A operação realizar a análise das unidades de medida do modelo matemático, visa relacionar a existência de conhecimento estável do estudante ou relacionar ideias que podem ser úteis na resolução do modelo (MENDOZA, 2009, p. 127).
} 
Terceira Ação: solucionar o modelo matemático. São as operações de aporte para a execução de encontrar a solução do problema a partir do modelo esboçado, então, selecionar o(s) método(s) matemático(s) para solucionar o modelo matemático, "é uma operação psíquica e motora; e a última operação desta ação é solucionar o modelo matemático, que requer essencialmente a organização psíquica dos procedimentos que serão aplicados" (MENDOZA, 2009, p. 80).

Quarta Ação: interpretar a solução. Para Mendoza (2009, p. 80), "as operações desta ação são essenciais para se observar o nível de compreensão do estudante, possibilitado através do esboço descritivo da forma de interpretação do resultado obtido da solução do modelo matemático". A operação de extrair os resultados significativos que tenham relação com o(s) objetivo(s) do problema, torna possível uma análise qualitativa da compreensão do processo. Dar resposta ao(s) objetivo(s) do problema, significa que o estudante realizou uma relação bem estabelecida dos dados para encontrar a resposta correta. Neste sentido,

\begin{abstract}
Realizar um informe baseado no(s) objetivo(s) do problema, é descrever as informações essenciais relacionadas ao objetivo do problema; e a última operação do processo é analisar a partir de novos dados e condições que tenham relação direta com o(s) objetivo(s) do problema(s), a possibilidade de reformular o problema, construir novamente o modelo matemático, solucionar o modelo matemático e interpretar a solução (MENDOZA, 2009, p.80).
\end{abstract}

Nesta proposta, o ponto de partida do ensino e aprendizagem de um conteúdo não é a definição, mas o problema, de forma que este expresse aspectoschave desse conteúdo, levando o aluno a ultrapassar o problema em si e a refletir sobre conceitos generalizados a que ele possa conduzi-lo. Ausubel et al. (1980), enfatiza que o enunciado do problema deve ser claro e significativo ao aluno e, para que se compreenda o problema, é preciso ser capaz de perceber o significado que suas proposições comunicam.

Conforme argumenta Pozo (1998, p. 33), "a solução de problemas começa com a ativação dos conhecimentos prévios dos estudantes, porém, ressalta-se que o problema deve ser significativo para o aluno". As proposições relativas ao problema consistem em aspectos relevantes do conhecimento prévio, as quais têm relação com o problema em questão, se os conhecimentos prévios existentes na estrutura cognitiva são claros, estáveis e diferenciáveis, facilitam na resolução de problemas, logo, deve-se averiguar os conhecimentos prévios dos estudantes.

Os conceitos aqui, foram abordados mediante a exploração de problemas, pois, o aluno não constrói um conceito em resposta a um problema, mas constrói um campo de conceitos que tomam sentido num campo de problemas. Um conceito matemático se constrói articulado com outros conceitos, por meio de uma série de retificações e generalizações.

Segundo Greca e Moreira (2003, p. 03), "o comportamento dos estudantes na resolução de problemas é guiado por hipóteses, analogias, metáforas, que dependem da conceitualização", neste sentido Vergnaud enfatiza que: 
A Figura 1 é o mapa conceitual da estratégia de resolução de problemas fundamentado na TAS.

Figura 1 - A estratégia de resolução de problemas, fundamentada na TAS

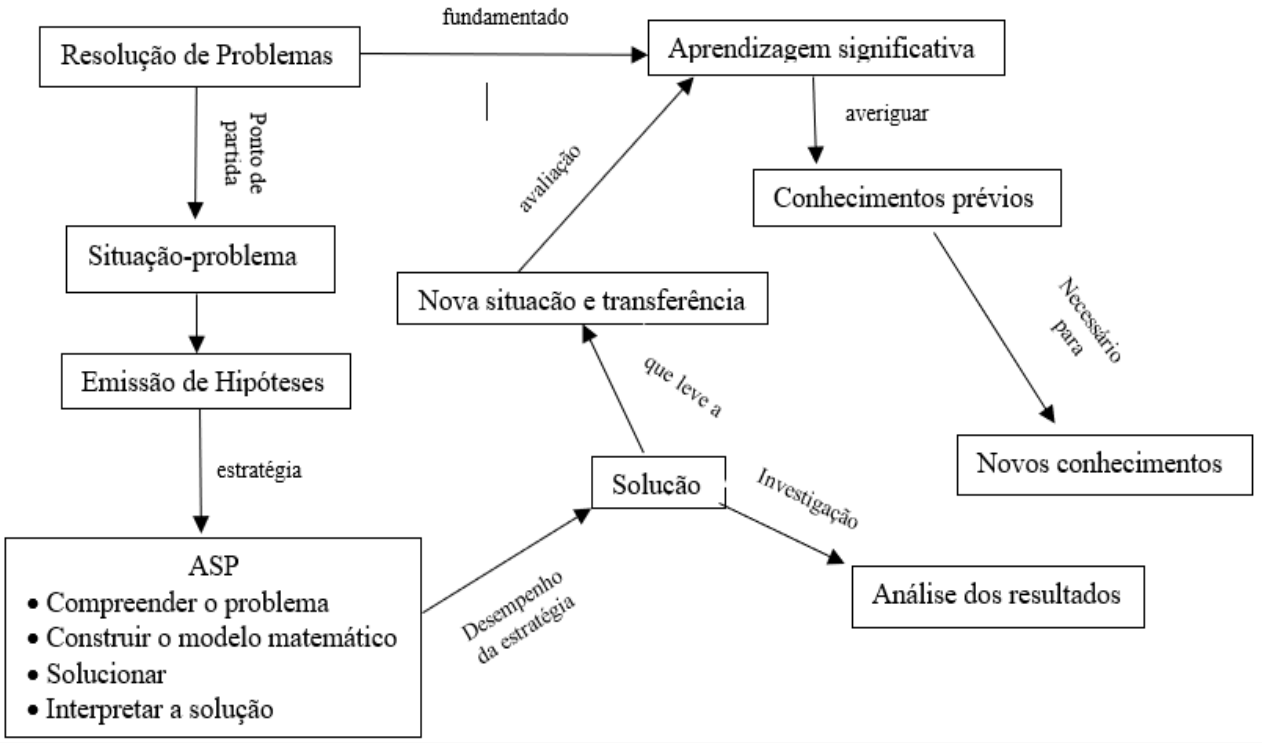

Fonte: Assunção (2019).

Sendo assim, a estratégia de resolução de problemas reflete a influência do tipo de problema envolvido e as condições nas quais a resolução de problema ocorre, assim como aspectos do funcionamento cognitivo do indivíduo.

\section{METODOLOGIA}

A pesquisa quantitativa procura "estudar os fenômenos de interesse da pesquisa em educação, geralmente através de estudos experimentais ou correlacionais, caracterizados primordialmente, por medições objetivas e análises quantitativas" (MOREIRA 2011, p.18).

O objetivo desta pesquisa foi analisar a efetividade da estratégia de resolução de problemas (ERP) como metodologia de ensino fundamentada na teoria da aprendizagem significativa de Ausubel. Para isso, 84 estudantes da 1o série do Ensino Médio do Colégio Militar CME-PMRR, distribuídos em quatro turmas, no turno vespertino, com idades entre 15 e 16 anos, foram submetidos a essa metodologia (grupo experimental) e a metodologia tradicional (grupo controle) para desenvolver os conteúdos de função e função afim. Do total da amostra, 43 estudantes (turmas B e D) fizeram parte do grupo experimental e o restante $(n=41)$ congregaram o grupo de controle (turmas $\mathrm{A}$ e $\mathrm{C}$ ).

Aos dois grupos do estudo (experimental e controle) foram aplicadas quatro provas de lápis e papel: i) duas provas antes do início da formação do conteúdo (_PRE) para avaliar o conhecimento prévio dos estudantes um pré-teste antes do início do conteúdo de funções e um pré-teste antes do início do conteúdo de funções afim; e ii) duas provas no final da formação do conteúdo para cada uma das metodologias (_POS) para avaliar a efetividade da metodologia ERP vis-a-vis a metodologia tradicional. Cada uma dessas duas provas refere-se ao conteúdo abordado: função e função afim; e apresentava três problemas que foram 
avaliados em quatro dimensões: 1) Compreender o problema; 2) Construir o modelo matemático; 3) Solucionar o modelo matemático; e 4) Interpretar a solução. Cada uma dessas dimensões foram avaliadas com escores de 1 a 5 , conforme instruções que constam no Quadro 2.

Quadro 2 - Avaliação qualitativa e quantitativa dos problemas das provas de lápis e papel sobre o conteúdo de função e funções afim

\section{Variável Independente X orientação do sistema de ações da Resolução de Problemas}

Variável Dependente Y Desempenho na resolução de problemas

Definição Conceitual: É a capacidade dos alunos de resolver problemas e fazer transferências para situações problema novas

Definição Operacional: É a diferença de desempenho comparando um ponto inicial com outro, a fim de resolver problemas e estabelecer transferências para situações problema novas.

\begin{tabular}{|c|c|}
\hline Dimensão & Descrição \\
\hline$Y_{1}$ & Desempenho de compreender o problema \\
\hline$Y_{2}$ & Desempenho de construir o modelo \\
\hline$Y_{3}$ & Desempenho de solucionar o modelo \\
\hline$Y_{4}$ & Desempenho de interpretar a solução \\
\hline
\end{tabular}

Medição: Para designar o resultado quantitativo a cada dimensão $\left(Y_{1}, Y_{2}, Y_{3}, Y_{4}\right)$ será utilizado uma escala de 1 até 5 pontos com o critério:

- Se todos os indicadores estão incorretos obterá a qualificação de um (1).

- Se o indicador essencial está incorreto ou parcialmente correto e/ou existe pelo menos outro indicador parcialmente correto obterá a qualificação de dois (2).

- Se o aluno tem somente correto o indicador essencial obterá a qualificação de três (3).

- Se o indicador essencial está correto, mas existe pelo menos outro indicador parcialmente correto obterá a qualificação de quatro (4).

- Se todos os indicadores estão corretos obterá a qualificação de cinco (5).

\begin{tabular}{|c|c|}
\hline Dimensão & Indicador \\
\hline$Y_{1}$ & $\begin{array}{l}\text { a) O aluno extrai os dados do problema? } \\
\text { b) O aluno determina as condições do problema? } \\
\text { c) O aluno define o(s) objetivo(s) do problema? }\end{array}$ \\
\hline$Y_{2}$ & $\begin{array}{l}\text { a) Determinar as variáveis e incógnitas. } \\
\text { b) Nominar as variáveis com suas características. } \\
\text { c) Construir o modelo matemático a partir das variáveis e condições. } \\
\text { d) Realizar análises das unidades de medidas do modelo matemático e } \\
\text { critério de aprovação. }\end{array}$ \\
\hline$Y_{3}$ & $\begin{array}{l}\text { a) Selecionar o(s) método(s) matemático(s) para solucionar o modelo } \\
\text { matemático. } \\
\text { b) Solucionar o modelo matemático. }\end{array}$ \\
\hline$Y_{4}$ & $\begin{array}{l}\text { a) Extrair os resultados significativos que tenham relação com o(s) } \\
\text { objetivo(s) do problema. } \\
\text { b) Dar resposta ao(s) objetivo(s) do problema. } \\
\text { c) Realizar um relatório baseado no(s) objetivo(s) do problema; Analisar a } \\
\text { partir de novos dados e condições que tenham relação direta ou não com } \\
\text { o(s) objetivo(s) do problema, a possibilidade de reformular o problema, } \\
\text { construir novamente o modelo matemático, solucionar o modelo } \\
\text { matemático e interpretar a solução. }\end{array}$ \\
\hline
\end{tabular}
Fonte: Assunção (2019). 
Nesse sentido, conforme podemos visualizar no quadro 2, existem quatro variáveis de interesse (dependentes), podendo ser criada uma quinta, que mensura $O$ desempenho geral do aluno no problema $\left(Y_{5}=Y_{1}+Y_{2}+Y_{3}+Y_{4}\right)$. $O$ desempenho do aluno em cada prova é mensurado pelo desempenho que ele obteve nos três problemas. Assim, podemos ter uma avaliação de desempenho $\left(Y_{n}\right)$, mensurada quantitativamente, antes (_PRE) e após (_POS) a formação do conteúdo, para cada um dos conteúdos lecionados (funções e funções afim) e no geral (funções + funções afim), segregada para cada um dos grupos do estudo (experimental versus controle) e no total. Dessa forma, as variáveis da pesquisa se resumem como proposto na Tabela 1.

Tabela 1 - Codificação das variáveis da Pesquisa

$\begin{array}{lc}\text { Código } & \text { Descrição } \\ \text { TURMA } & \text { Turma do aluno (A, B, C ou D) } \\ \text { GRUPO } & \text { Grupo experimental ou controle } \\ \text { CONTEUDO } & \text { Funções e função afim } \\ \text { Y1_PRE } & \text { Compreender o problema (Pré-teste) } \\ \text { Y2_PRE } & \text { Construir o modelo matemático (Pré-teste) } \\ \text { Y3_PRE } & \text { Solucionar o modelo matemático (Pré-teste) } \\ \text { Y4_PRE } & \text { Interpretar a solução (Pré-teste) } \\ \text { Y1_POS } & \text { Compreender o problema (Pós-teste) } \\ \text { Y2_POS } & \text { Construir o modelo matemático (Pós-teste) } \\ \text { Y3_POS } & \text { Solucionar o modelo matemático (Pós-teste) } \\ \text { Y4_POS } & \text { Interpretar a solução (Pós-teste) } \\ \text { Y5_PRE } & \text { Escore geral (Pré-teste) } \\ \text { Y5_POS } & \text { Escore geral (Pós-teste) }\end{array}$

Fonte: Assunção (2019).

\section{ANÁLISE DOS DADOS}

Inicialmente, analisou-se a fidedignidade do instrumento de medida $\left(Y_{n}\right)$. Segundo (PASQUALI, 2010), a fidedignidade se refere a quanto os escores de um sujeito se mantêm idênticos em ocasiões diferentes. Essa ocorrência evidentemente supõe que o traço que o teste mede se mantenha constante sobre diferentes ocasiões. "Assim, o conceito de fidedignidade, na verdade, se refere ao quanto o escore obtido no teste se aproxima do escore verdadeiro do sujeito em um traço qualquer" (PASQUALI, p. 194, 2010).

Na presente pesquisa, como tem-se o teste diagnóstico antes (_PRE) e após (_POS) a formação do conteúdo é possível calcular a precisão Teste-reteste para cada um dos conteúdos, assim como, a partir do conjunto de itens (problemas em cada uma das dimensões) dos testes, computar a Consistência interna (Alfa de Cronbach) do instrumento em cada um dos conteúdos. Nesse último caso basta o cálculo do Alfa de Cronbach para o teste de diagnóstico antes (_PRE) da formação do conteúdo ${ }^{1}$.

Num segundo momento, iniciou-se uma análise descritiva, essencialmente, pela visualização dos escores das variáveis $Y_{n}$ através de gráficos de barras agrupados pelos testes de diagnósticos antes/depois e os grupos 
controle/experimental. Assim, em termos preliminares, ter-se-ia uma discussão da distribuição dos escores entre os grupos e testes diagnósticos e alguns indícios sobre a direção da média dos escores (maior/menor).

Para os escores serem comparáveis entre os conteúdos, transformou-os para a base 100 tendo como limite inferior o valor zero ${ }^{2}$, ou seja, cada escore $\left(Y_{n}\right)$ passou a ser um "coeficiente de rendimento". Por exemplo, um escore de 75 indica que o aluno obteve $75 \%$ dos "pontos" distribuídos no teste (pré ou pós), no conteúdo (funções, funções afim, ou todo conteúdo) ou na dimensão $\left(Y_{n}\right)$. Esses escores padronizados na base 100 permaneceram nas análises seguintes ${ }^{3}$.

Finalmente, em termos inferenciais, no mínimo três abordagens poderiam ser adotadas: 1) computar as diferenças entre os escores dos testes após e antes a formação do conteúdo $\left(\mathrm{Y}_{\mathrm{n}} \mathrm{POS}-\mathrm{Y}_{\mathrm{n}} \mathrm{PRE}\right)$ e aplicar testes de médias entre os dois grupos (controle e experimental) para cada um dos conteúdos e/ou todo o conteúdo; 2) considerar os conteúdos simultaneamente como amostra repetida de um mesmo indivíduo e rodar cinco modelos linear generalizado misto - GLMM - (um para cada dimensão $Y_{n}$ ) com efeitos aleatórios (nas turmas, por exemplo) tendo a diferença dos escores $\left(\mathrm{Y}_{\mathrm{n}} \mathrm{POS}-\mathrm{Y}_{\mathrm{n}} \mathrm{PRE}\right)$ como variável dependente $\mathrm{e}$ conteúdo e grupo como fatores fixos ${ }^{4}$; ou 3 ) cinco regressões lineares múltiplas, uma para cada $Y_{n} \_P O S$ como variável dependente, em cada um dos conteúdos ou todo conteúdo, com a variável grupo como fator (variável binária com 0 para o grupo controle e 1 para o grupo experimental) e a $Y_{n_{2}}$ PRE como covariável.

Optou-se pela última abordagem em detrimento da abordagem mais simples do teste de médias, pois quando se executam pré e pós-testes deseja-se controlar ou remover o efeito do pré-teste para poder perceber possíveis alterações devido a intervenção feita. A utilização de diferenças de escores não permite isso, uma vez que o pré-teste está geralmente correlacionado ao resultado (diferença) e assim a variação nos valores do pré-teste não é removida (DANCEY, REIDY, p. 435, 2013). Em geral, a ANCOVA ${ }^{5}$ tende a fornecer um teste mais poderoso de hipóteses do que a diferença de escores. Optou-se pela última abordagem em detrimento da segunda abordagem (GLMM), pois nessa abordagem mais complexa ter-se-ia os resultados agregados e na ANCOVA a possibilidade de detalhar os resultados por conteúdo ${ }^{6}$.

Assim, os $\mathrm{k}$ modelos estimados foram:

$$
Y_{n} P O_{i k}=\alpha_{k}+\beta_{1 k} G R U P O_{i k}+\beta_{2 k} Y_{n} P R E_{i k}+u_{i k}
$$

Onde, $Y_{n}$ POS representa o escore padronizado na base 100 no pós-teste do aluno i no conteúdo $k$ (funções, funções afim ou todo conteúdo); GRUPO é variável dummy que capta o efeito diferencial do grupo experimental (GRUPO $=0$ se controle e GRUPO = 1 se experimental); $Y_{n}$ PRE é a variável que busca controlar os efeitos diferenciais individuais entre os grupos (controle versus experimental) através dos escores padronizados na base 100 do pré-teste; $\alpha$ é a constante/intercepto de cada modelo k; $\beta_{1}$ e $\beta_{2}$ são os coeficientes/parâmetros estimados no modelo $k$ para os efeitos marginais do grupo experimental e uma unidade adicional no escore do pré-teste, respectivamente; $u_{\mathrm{ik}}$ representa os resíduos, para cada aluno i, nas regressões k, onde não se assume homogeneidade nem normalidade, pois os erros-padrão (IC $=95 \%)$ dos parâmetros da regressão foram estimados por bootstrap $(n=1.000)$, com variâncias estratificadas pela variável TURMA e corrigidos para viés $(\mathrm{Bca})^{7}$. 


\subsection{FIDEDIGNIDADE DO INSTRUMENTO}

\section{Correlações entre os testes diagnósticos}

De uma forma geral, as correlações entre os testes diagnósticos (pré versus pós) apresentaram magnitudes elevadas $(\rho>0,50)$ e foram altamente significativas ( $p$-valor $<0,000$ ). Quando se considera todos os conteúdos (Geral) ou as somas dos escores (Y5) os valores dos coeficientes de correlação estimados mostram-se maiores. Esses indícios atestam a fidedignidade do instrumento de avaliação conforme a tabela 2 .

Tabela 2 - Correlações de Spearman ( $\rho)$ entre os pós e pré-teste por conteúdo e geral $(n=84)$

\begin{tabular}{cccccc} 
CONTEUDO & Y1 & Y2 & Y3 & Y4 & Y5 \\
Funcão & $.563^{* *}$ & $.715^{* *}$ & $.733^{* *}$ & $.528^{* *}$ & $.759^{* *}$ \\
Função afim & $.683^{* *}$ & $.816^{* *}$ & $.777^{* *}$ & $.664^{* *}$ & $.785^{* *}$ \\
Geral & $.708^{* *}$ & $.825^{* *}$ & $.814^{* *}$ & $.676^{* *}$ & $.826^{* *}$ \\
\hline & $* *$ A correlação é significativa no nível 0,01 (bilateral) &
\end{tabular}

Fonte: Assunção (2019).

\subsection{ALFA DE CRONBACH DOS PRÉ-TESTES}

Os resultados dos alfas de Cronbach $(\alpha)$ apresentados na Tabela 3 atestam os achados apresentados na seção anterior, corroborando assim, a fidedignidade do instrumento. Note que a maioria dos valores dos $\alpha$ 's foram maiores que 0,70 ou ao redor desse valor ${ }^{8}$. Mais uma vez, a medida para o escore geral (Y5) e todo conteúdo (Geral) foram as mais promissoras (maiores). Na verdade, de um modo geral, o instrumento mostrou-se confiável (fidedigno), com boas medidas de precisão, sendo que todas as dimensões merecem/podem ser avaliadas individualmente.

Tabela 3 - Alfa de Cronbach $(\alpha)$ do pré-teste por conteúdo e geral $(n=84)$

\begin{tabular}{cccccc} 
CONTEUDO & Y1 & Y2 & Y3 & Y4 & Y5 \\
Funcão & 0,686 & 0,742 & 0,763 & 0,675 & 0,914 \\
Função afim & 0,694 & 0,785 & 0,768 & 0,711 & 0,899 \\
Geral & 0,815 & 0,841 & 0,814 & 0,736 & 0,942 \\
\hline
\end{tabular}

Fonte: Assunção (2019).

\subsection{REGRESSÕES MÚLTIPLAS (ANCOVA)}

As tabelas seguintes resumem as principais estatísticas de adequação dos modelos estimados considerando todo o conteúdo para cada uma das dimensões. De uma forma geral, todos os modelos apresentaram excelente ajuste e corroboraram o melhor aproveitamento (maior coeficiente de rendimento) do grupo experimental mesmo depois de controlarmos pelo primeiro teste 
diagnóstico (pré-teste). Para exemplificar discutir-se-á os resultados para todo o conteúdo (funções + funções afim) e o escore geral (Y5).

\subsection{TODO O CONTEÚDO (GERAL) E ESCORE TOTAL (Y5)}

Conforme Tabela 4, o ajuste do modelo mostrou-se excelente: i) $R^{2}=0,847$, indicando que $84,7 \%$ da variabilidade dos escores pós-teste são explicados pela variabilidade dos escores pré-teste e o fato do aluno pertencer ao grupo de controle ou experimental ${ }^{9}$; e ii) a estatística de Durbin-Watson ao redor de 2 indica que o modelo não incorre em problemas de especificação ${ }^{10}$. A Tabela 5 complementa a análise anterior ao evidenciar um valor muito alto para o teste $F$, altamente significativo ( $p$-valor $<0,000$ ), de forma a comprovar que, no conjunto, as variáveis Y5_PRE e GRUPO são significativas para explicar a variável Y5_POS.

O coeficiente (B) na Tabela 6 para a variável GRUPO indica que os alunos do grupo experimental $(G R U P O=1)$ obtiveram um aproveitamento 9,374 pontos percentuais maior que os alunos do grupo controle no pós-teste, permanecendo $o$ aproveitamento no pré-teste constante. Essa diferença foi altamente significativa $(p \text {-valor }<0,000)^{11}$. Por exemplo, um aluno que obteve $75 \%$ de aproveitamento na primeira prova, se ele é do grupo experimental, sua estimativa de desempenho na segunda prova é $80,772 \%(=12,298+9,374 \times 1+0,788 \times 75)$. Se o aluno for do grupo de controle sua estimativa de aproveitamento na segunda prova será de $71,398 \%$ $(=12,298+9,374 \times 0+0,788 \times 75)$.

Tabela 4 - Sumarização dos modelos considerando todo o conteúdo (Geral)

\begin{tabular}{cccccc} 
Modelo & R & R quadrado & $\begin{array}{c}\text { R quadrado } \\
\text { ajustado }\end{array}$ & $\begin{array}{c}\text { Erro padrão } \\
\text { da estimativa }\end{array}$ & $\begin{array}{c}\text { Durbin- } \\
\text { Watson }\end{array}$ \\
Y5_POS & $.920^{\mathrm{a}}$ & 0,847 & 0,843 & 4,70527 & 1,980 \\
Y1_POS & $.803^{\mathrm{a}}$ & 0,644 & 0,635 & 5,69800 & 2,076 \\
Y2_POS & $.876^{\mathrm{a}}$ & 0,768 & 0,762 & 5,17594 & 1,668 \\
Y3_POS & $.900^{\mathrm{a}}$ & 0,810 & 0,806 & 6,06701 & 1,740 \\
Y4_POS & $.874^{\mathrm{a}}$ & 0,764 & 0,758 & 8,33084 & 1,674 \\
\hline
\end{tabular}

a. Preditores: (Constante), Yn_PRE, GRUPO

b. Variável Dependente: Yn POS

Fonte: Assunção (2019).

Tabela 5 - ANOVA dos modelos considerando todo o conteúdo (Geral)

\begin{tabular}{|c|c|c|c|c|c|c|}
\hline Modelo & & $\begin{array}{l}\text { Soma dos } \\
\text { Quadrados }\end{array}$ & GI & $\begin{array}{l}\text { Quadrado } \\
\text { Médio }\end{array}$ & $\mathbf{F}$ & p-valor \\
\hline \multirow{3}{*}{ Y5_POS } & Regressão & 9923,948 & 2 & 4961,974 & 224,123 & $.000^{\mathrm{b}}$ \\
\hline & Resíduo & 1793,304 & 81 & 22,140 & & \\
\hline & Total & 11717,252 & 83 & & & \\
\hline \multirow{3}{*}{ Y1_POS } & Regressão & 4760,666 & 2 & 2380,333 & 73,315 & $.000^{b}$ \\
\hline & Resíduo & 2629,839 & 81 & 32,467 & & \\
\hline & Total & 7390,506 & 83 & & & \\
\hline
\end{tabular}




\begin{tabular}{ccccccc}
\hline & Regressão & 7166,677 & 2 & 3583,338 & 133,755 & $.000^{\text {b }}$ \\
Y2_POS & Resíduo & 2170,019 & 81 & 26,790 & & \\
& Total & 9336,695 & 83 & & & \\
\hline \multirow{2}{*}{ Y3_POS } & Regressão & 12736,008 & 2 & 6368,004 & 173,003 & $.000^{\text {b }}$ \\
& Resíduo & 2981,493 & 81 & 36,809 & & \\
& Total & 15717,501 & 83 & & & \\
Y4_POS & Regressão & 18228,586 & 2 & 9114,293 & 131,324 & $.000^{\text {b }}$ \\
& Resíduo & 5621,631 & 81 & 69,403 & & \\
& Total & 23850,217 & 83 & & &
\end{tabular}

a. Variável Dependente: Yn_POS

b. Preditores: (Constante), Yn_PRE, GRUPO

Fonte: Assunção (2019).

Tabela 6 - Coeficientes dos modelos considerando todo conteúdo (Geral)

\begin{tabular}{|c|c|c|c|c|c|c|c|c|}
\hline \multirow{2}{*}{$Y$} & \multirow{2}{*}{$x$} & \multicolumn{2}{|c|}{$\begin{array}{c}\text { Coeficientes não } \\
\text { padronizados }\end{array}$} & \multirow[t]{2}{*}{$\begin{array}{l}\text { Coeficientes } \\
\text { padronizados }\end{array}$} & \multirow{2}{*}{$T$} & \multirow{2}{*}{$\begin{array}{c}p- \\
\text { valor }\end{array}$} & \multicolumn{2}{|c|}{$\begin{array}{l}95 \% \text { Intervalo de } \\
\text { Confiança para B }\end{array}$} \\
\hline & & B & $\begin{array}{c}\text { Erro } \\
\text { Padrão }\end{array}$ & & & & $\begin{array}{l}\text { Limite } \\
\text { inferior }\end{array}$ & $\begin{array}{l}\text { Limite } \\
\text { superior }\end{array}$ \\
\hline \multirow{3}{*}{ Y5_POS } & (Constante) & 12,298 & 3,736 & & 3,292 & 0,001 & 4,864 & 19,733 \\
\hline & GRUPO & 9,374 & 1,078 & 0,397 & 8,697 & 0,000 & 7,229 & 11,518 \\
\hline & Y5_PRE & 0,788 & 0,050 & 0,719 & 15,756 & 0,000 & 0,688 & 0,887 \\
\hline \multirow{3}{*}{ Y1_POS } & (Constante) & 32,416 & 5,397 & & 6,007 & 0,000 & 21,678 & 43,153 \\
\hline & GRUPO & 5,508 & 1,307 & 0,294 & 4,216 & 0,000 & 2,909 & 8,108 \\
\hline & Y1_PRE & 0,649 & 0,068 & 0,662 & 9,515 & 0,000 & 0,513 & 0,785 \\
\hline \multirow{3}{*}{ Y2_POS } & (Constante) & 20,706 & 4,008 & & 5,166 & 0,000 & 12,730 & 28,681 \\
\hline & GRUPO & 5,386 & 1,172 & 0,255 & 4,594 & 0,000 & 3,053 & 7,719 \\
\hline & Y2_PRE & 0,721 & 0,052 & 0,773 & 13,898 & 0,000 & 0,618 & 0,824 \\
\hline \multirow{3}{*}{ Y3_POS } & (Constante) & 7,365 & 4,062 & & 1,813 & 0,074 & $-0,718$ & 15,448 \\
\hline & GRUPO & 9,430 & 1,373 & 0,345 & 6,867 & 0,000 & 6,698 & 12,162 \\
\hline & Y3_PRE & 0,811 & 0,055 & 0,746 & 14,858 & 0,000 & 0,703 & 0,920 \\
\hline \multirow{3}{*}{ Y4_POS } & (Constante) & 9,532 & 5,165 & & 1,846 & 0,069 & $-0,745$ & 19,809 \\
\hline & GRUPO & 18,986 & 1,905 & 0,563 & 9,968 & 0,000 & 15,196 & 22,775 \\
\hline & Y4_PRE & 0,688 & 0,075 & 0,522 & 9,234 & 0,000 & 0,540 & 0,836 \\
\hline
\end{tabular}

Fonte: Assunção (2019).

\section{DISCUSSÃO DOS RESULTADOS ESTATÍSTICOS}

A dificuldade para definir de forma precisa o conceito de função é apontada por Meneghetti e Redling (2012), como obstáculo para a compreensão de outros conceitos relacionados, e pode ser superado a partir do desenvolvimento de tarefas de aprendizagem sequenciais mais elaboradas, que permitam enxergar a 
necessidade dos alunos em dominar o conteúdo, e assim, alcançar êxito na resolução dos problemas e na aprendizagem significativa.

No entanto, Schonardie (2011), constatou que apesar dos alunos conseguirem montar o gráfico, tiveram dificuldades para explicá-los. Apesar disso, a autora avalia positivamente o uso da modelagem matemática, pois essa metodologia possibilita melhor compreensão de função afim, a partir da contextualização do conteúdo.

Mas, também em outro estudo acerca da temática (COSTA, 2010), verificou que os resultados do pré-teste de sua pesquisa, corroboraram baixo desempenho dos alunos na construção dos gráficos, na determinação da expressão algébrica, formulação da lei de uma função partindo de uma situação problema e compreensão do que sejam variáveis dependente e independente, porém, após a intervenção os alunos desenvolveram habilidades para resolver problemas e apropriaram-se de conceitos matemáticos.

Segundo Mendoza (2009), "a resolução de problema exige certa dose de iniciativa e criatividade, aliada ao conhecimento de algumas estratégias". Através dos resultados da avaliação diagnóstica, identificou-se que os estudantes alcançaram índice satisfatório nas habilidades para resolver problemas relacionados a noções básicas de função, porém observou-se que resolveram os problemas sem saber que se tratava de noções de função.

Segundo Talízina (1988, p. 47), "para se desvendar os mecanismos internos que caracterizam a atividade cognoscitiva não é suficiente verificar a capacidade de resolver determinadas situações-problema, pois a obtenção de uma resposta correta não significa necessariamente raciocínio correto". Isso significa que alguns estudantes conseguem resolver corretamente a situação-problema, porém, não tem consciência do porquê, nem sabem muito bem explicar como.

As dificuldades encontradas pelos alunos, provavelmente, devem ocorrer ao transpor conhecimentos da Aritmética para a Álgebra, pois, muitas vezes, no estudo das funções é contemplado apenas o aspecto algébrico, desse modo são negligenciados diferentes tipos de representação de forma intrínseca contemplados no estudo de função. Além disso, considera-se também, a falta de conhecimentos básicos para o entendimento de um novo conceito.

Portanto, para a maioria das dimensões analisadas não foi observada aprendizagem significativa no grupo controle, dado que as novas informações não interagiram com a estrutura do conhecimento - os subsunçores, ou seja, informações que os alunos já possuem (AUSUBEL, et al. 1980). Os alunos do grupo controle apenas memorizaram os conteúdos, eles relacionaram o conhecimento de forma arbitrária, e desse modo não adquiriram autonomia para agir na sua realidade.

Meneghetl e Redling, (2012, p. 78), enfatizam que "a intervenção educativa deve voltar-se ao saber fazer, ao aprender a aprender, devendo ser coerente com o nível de desenvolvimento dos alunos". Sendo assim, é necessário realizar o diagnóstico dos conceitos prévios dos estudantes, pois leva a compreensão de quais conceitos formais o professor precisa abordar ao ensinar sobre função. Nesse sentido, tarefas de aprendizagem mais elaboradas, que demande domínio dos conceitos anteriores para transferência de conhecimento de uma situaçãoproblema para outra, contribuem para aprendizagem significativa. 
Contudo, junto à resolução de problemas, não se pode ignorar as possibilidades e os recursos disponíveis para compreender funções. "Do ponto de vista da tecnologia disponível para os alunos, quando se contempla sua relevância para o ensino, alguns estudiosos destacam melhorias no uso da modalidade Blended Learning no ensino médio, que combina aulas presenciais com recursos digitais" (CONDIE; LIVINGSTON, 2007, p. 84).

Ao se apropriar desta modalidade mista no ensino de função afim, verifica-se efetividade no processo ensino aprendizagem de resolução de problemas deste conteúdo, pois facilita a compreensão de um conceito abstrato como é a definição de função afim, além disso amplia a participação do aluno, pois o acesso as informações ultrapassa a sala de aula, porém destaca-se como limitações, pouca infraestrutura voltada a informática na escola, baixa adesão de professores e sobrecarga de trabalho docente.

\subsection{EFETIVIDADE DA SEQUÊNCIA DIDÁTICA UTILIZANDO A ESTRATÉGIA DE RESOLUÇÃO DE PROBLEMA}

Quanto a aprendizagem dos conteúdos trabalhados, no que se refere a todas as dimensões: compreensão do problema (Y1), construção do modelo (Y2), solução do modelo (Y3), interpretação da solução (Y4), considera-se que a intervenção com uma abordagem diferente da tradicional favoreceu a aprendizagem significativa dos conteúdos trabalhados, o que foi reafirmado de forma evidente nos dados da estatística descritiva, pelas médias e desvios padrão dos escores.

"As atividades propostas e os testes diagnósticos com resolução de problemas favoreceram aprendizagem satisfatória dos conteúdos, pois os estudantes adquiriam motivação e independência para solucionar os problemas" (BOSCHETTO, 2015, p. 75), corroborando com os resultados do estudo da autora, que abrangeu conceitos sobre função afim, realizado com alunos do primeiro ano do ensino médio.

Para Mendonza (2009, p. 90), "a resolução de um problema atua como organizador do processo de ensino de matemática, portanto, ao ser elaborado precisa ser considerado o estado psicológico, a motivação dos alunos, o que requer prudência na atividade docente". Ausubel et al. (1980) acrescenta o conhecimento prévio como fator primordial para aprendizagem, e da mesma forma, observa o papel do professor no sentido de considerar esse conhecimento nas estratégias metodológicas ao inserir um novo conhecimento.

Com relação a função afim, Boschetto (2015) obteve resultado diferente do apresentado nesta pesquisa, ao associar a resolução de problemas às mídias informatizadas, essa combinação aumentou o rendimento dos alunos, que adquiriram autonomia e mostraram-se mais motivados, favorecendo a aprendizagem do conteúdo por meio da experimentação realizada com as tecnologias e atuação do professor.

Quando os dados foram submetidos a ANCOVA, mais uma vez os resultados corroboraram o melhor aproveitamento (maior coeficiente de rendimento) do grupo experimental mesmo depois de controlarmos pelo primeiro teste diagnóstico (pré-teste). Um outro fator a ser considerado, é a necessidade do docente atentar para questões da realidade do aluno ao ensinar matemática, 
desse modo os alunos terão mais facilidade para entendimento dos conteúdos, sendo essa interação essencial para qualquer aprendizado de qualidade.

Nesse seguimento, vale evidenciar a discussão de Feltes e Puhk (2016), quando apresentam suas observações durante o ensino de função, atribuindo-lhe a etapa levar para a vida, que significa a representação pelos alunos de uma situação cotidiana ainda não abordada na sala de aula, de tal forma, ocorria o estudo do gráfico da função, segundo os autores pode-se afirmar, que desse modo é possível dar sentido ao que estão aprendendo a partir de situações de suas próprias vivências, a maioria dos alunos mostraram-se mais participativos e curiosos, souberam realizar a proposta matemática e também relacionar com situações do cotidiano.

\section{CONSIDERAÇÕES FINAIS}

A aprendizagem mecânica tem desconsiderado e negligenciado o conhecimento prévio do estudante, porém, em uma perspectiva ausubeliana, os novos conhecimentos, só serão substantivamente assimilados e armazenados se forem consideradas as ideias preexistentes na estrutura cognitiva do estudante.

Analisando o resultado de modo geral, observou que alguns estudantes de ambos os grupos tinham noções básicas do conceito de função, porém eles apresentaram dificuldades em estabelecer relação com os conceitos de função, e não utilizavam da linguagem matemática. De início a maioria dos estudantes respondiam os problemas por ensaio e erro, não escreviam a lei de formação da função e não escreviam a expressão $f(x)$. Outra dificuldade eloquente dos alunos, apresentou-se no momento de justificar sua resposta, os estudantes não utilizavam da linguagem matemática adequada a cada situação, o que demonstrou a falta de hábito dos estudantes em trabalhar com problemas que os façam expressar e escrever o raciocínio.

Observou também, que os estudantes dos dois grupos cometiam erros na solução, ou estava relacionado as quatro operações ou quando a solução envolvia outros conhecimentos matemáticos para resolvê-los na qual os estudantes não lembravam ou não dominavam.

No entanto, houve uma melhora gradativa destas habilidades dos estudantes do grupo experimental no decorrer da proposta pedagógica, oportunizada pelas atividades de interpretação e análise gráfica, construir gráfico a partir de situações problemas, analisar gráficos no qual a situação envolvia outra área de conhecimento, sendo que estas atividades proporcionaram o envolvimento dos alunos em discussões e reflexões sobre as diferentes representações de uma função e os induziram a expor seu pensamento e escrevê-lo, oportunizando ao professor visualizar as possíveis falhas de interpretação por parte dos alunos e seus conhecimentos prévios acerca do tema em estudo, assim como, ajudá-los a expressarem-se de forma clara usando termos matemáticos, ampliando seus conhecimentos em linguagem matemática.

Já os estudantes do grupo controle tiveram mais dificuldades, pois eles realizavam alguns problemas apenas como exercício no final do conteúdo, através de aulas expositivas. Um dos estudantes disse: "professora passa qualquer função aí que sei responder/calcular, agora quando vem com problemas dá um nó na cabeça e não sei nem por onde começar. 
Diante dos resultados apresentados, conclui-se que a instrução centrada na resolução de problema é mais eficiente que o ensino tradicional, onde os problemas são aplicações ao final de cada tema de estudo, logo a teoria da aprendizagem significativa associada a resolução de problemas, demonstrou potencial significativo ao comparar os resultados obtidos entre o grupo experimental e o grupo controle. 
PROBLEM-SOLVING AS A

METHODOLOGICAL TEACHING STRATEGY

BASED ON THE THEORY OF MEANINGFUL

LEARNING: A QUANTITATIVE ANALYSIS

\begin{abstract}
Nowadays, in the scientific field, there is an essential discussion aiming at analyzing the effectiveness of the problem-solving strategy as a teaching methodology based on the meaningful learning theory of Ausubel. The investigation subjects were 84 students enrolled in the 1st year of a military high school from PMRR, distributed among four different classes that comprised an experimental and a control group. During such lessons, content functions and related functions were developed. The results were analyzed based on the descriptive statistics and ANCOVA to evaluate the effectiveness of the problem -solving methodology based on the theory of meaningful learning versus the use of a traditional methodology. It could be noticed that the learning of the contents in the experimental group after the intervention seemed to present a better performance in the tests than the control group.
\end{abstract}

KEYWORDS: Meaningful Learning. Problem-solving. Concept of Function. 
1 Como veremos adiante, os pré-testes serviram como variável controle (X) nas regressões lineares executadas e os pós-testes ( $Y n)$ foram as variáveis dependentes. Se houver erros de medidas na variável dependente não teremos muitos problemas (perca de eficiência), no entanto, caso haja erros de medidas nos $X^{\prime}$ 's pode-se-ia incorrer em vieses. Por isso, um cuidado maior com a mensuração das variáveis independentes.

2 De cada escore subtrai-se o mínimo possível de obter no conteúdo/teste/dimensão e divide-se o valor pelo máximo de se obter menos o mínimo, e depois, multiplica 100: \{[ (Escore - MínEscore) / (MáxEscore MínEscore)] x 100\}. Por exemplo, em qualquer dimensão, por teste e conteúdo, o máximo que o aluno pode tirar é um escore de 15 ( $5 \times 3$ problemas) e o mínimo um escore de 3 ( $1 \times 3$ problemas), assim, se o aluno tirou um escore de 12 ele obteve um aproveitamento de $75 \%=\{[(12-3) /(15-3)] \times 100\}$.

3 Como a transformação é linear os resultados estatísticos não mudariam caso optasse pelos escores originais. A padronização é apenas uma questão de conveniência e interpretação.

4 Ou Yn_POS como variável dependente e conteúdo e grupo como fatores fixos e a variável Yn_PRE como covariável.

$5 \mathrm{~A}$ abordagem três também é conhecida na literatura como ANCOVA (Análise de Covariância).

$6 \mathrm{Na}$ verdade, executamos as três abordagens e os resultados foram os mesmos. Isso será fácil de constatar adiante, pois as significâncias (rejeição das hipóteses de igualdade entre os grupos) foram altas o suficiente ao ponto de não mudar dependendo da abordagem utilizada.

7 Independentemente se assumíssemos a hipótese de normalidade e homogeneidade dos resíduos os resultados seriam os mesmos, uma vez que o teste de Levene não rejeitou a homogeneidade em todos os modelos e, apesar de não se verificar normalidade na maioria dos modelos, pelo teste Shapiro-Wilk, ela não foi exacerbada ao ponto de colocar em cheque os testes de hipóteses. Mais uma vez, os altos valores das significâncias (estatística t dos parâmetros dos modelos estimados) corroboram esse argumento.

8 A literatura indica que temos que ter um $\alpha>0,70$, no entanto, em pesquisas exploratórias aceita-se valores maiores que 0,60 (Pasquali, 2010).

9 R na Tabela 3 indica a correlação de Pearson entre os valores previstos e observados.

10 Ou seja, parace que realmente a amostra é i.d.d. e não existe correlação entre as observações.

11 Nesse caso deve-se olhar para os p-valor da Tabela 25, pois é nela que se evidencia os erros-padrão computados via Bootstrap $(n=1.000)$, corrigidos e acelerados por viés e estratificado pela variável TURMA. Note que, mesmo assumindo normalidade e/ou homogeneidade, ou seja, considerando os errospadrão/t estatístico/p-valor da Tabela 24, os resultados não mudariam.100\%. 


\section{REFERÊNCIAS}

ASSUNÇÃO, J. A. Proposta, implementação e avaliação de uma metodología de ensino no conteúdo de função, utilizando uma estratégia de resolução de problemas fundamentada na teoría da aprendizagem significativa de Ausubel. Tese (Doutorado em Educação) - Universidade de Burgos (UBU), Espanha, 2019.

AUSUBEL, D. P. Aquisição e retenção do conhecimento: uma perspectiva cognitiva. Tradução Lígia Teopisto. Lisboa: Plátano. 2003.

AUSUBEL, D. P.; NOVAK, J. D.; HANESIAN, H. Psicologia educacional. Tradução Eva Nick. Rio de Janeiro: 2 ed. Melhoramentos. 1980.

BOSCHETTO, V. C. Função afim e suas propriedades através da resolução de problemas (Dissertação de Mestrado). Universidade Estadual Paulista -UNESP. São José do Rio Preto, SP, Brasi, 2015.

CONDIE, R.; LIVINGTON, K. Blending online learning with tradiciona approaches: changing pratices. British Journal od Educational Technology, V. 38, n. 2, p. 337348, 2007.

COSTA, S.S. Função Afim: Resolução de Problemas - Mídias. Porto Alegre, 2010. Universidade Federal do Rio Grande do Sul (Monografia).

COSTA, S. S. C. $O$ aprender pela resolução de problemas. In: MASINI, E. F. S.; MOREIRA, M. A. Aprendizagem significativa: condições para ocorrência e lacunas que levam a comprometimento. São Paulo: Vetor, 2008.

DANCEY, C. P., \& Reidy, J. (2013). Estatística sem matemática para psicologia (5th ed.). Porto Alegre: Penso.

DANTE, L. R. Tudo é Matemática. São Paulo: Ática, 2008.

FELTS, C.M.; PUHK, C.S. Gráfico da Função Quadrática: Uma proposta de ensino potencialmente significativa. Scientia Kun Indústria, v.4, n.4, 202-206, 2016.

GRECA, I. M.; MOREIRA, M. A. Do saber fazer ao saber dizer: uma análise do papel da resolução de problemas na aprendizagem conceitual de Física. Ensaio, Belo Horizonte, v. 5, n. 1, p. 52-67, 2003. aprendizaje de los alunos en la actividad de situaciones problema en 
matemática en la asignatura de álgebra lineal, en el contexto de la Faculdade Actual de la Amazônia. Tese (Doutorado em Psicopedagogia) - Universidade de Jaén (UJAEN), Espanha, 2009.

MENEGHETTI, R. C. G.; Redling, J. P. (2012). Tarefas Alternativas para o Ensino e a Aprendizagem de Funções: análise de uma intervenção no ensino médio. Bolema, Rio Claro (SP), v. 26, n. 42A, p. 193-229.

MOREIRA, M. A. A Teoria da Aprendizagem Significativa e sua implementação em sala de aula. Brasília: UnB, 2006.

MOREIRA, M. A. Metodologias de pesquisa em ensino. São Paulo: Ed. Livraria da Física, 2011.

PASQUALI, L. (2010). Testes referentes a constructo: teoria e modelo de construção. (L. Pasquali, Ed.)Instrumentação psicológica: fundamentos e prática. Porto Alegre: Artmed.

POLYA, G.A. A arte de resolver problemas. Rio de Janeiro: Interciência, 1995.

POZO, J. I. (1998). A solução de problemas: aprender a resolver, resolver para aprende. Porto Alegre: Artmed.

SCHONARDIE, B. (2011). Modelagem matemática e introdução da função afim no ensino fundamental (Dissertação de mestrado). Universidade Federal do Rio Grande do Sul-UFRGS. Porto Alegre, RS, Brasil.

STERnBERG, R.J. Psicologia Cognitiva. 5. ed. São Paulo: Cengage Learnig, 2010.

TALÍzINA, Nina. Psicologia do Ensino. Moscou: Progresso, 1988.

VERGNAUD, G. La Théorie des champs conceptuels: Recherches em Didactique des Mathématiques, 10(23), p.133-170. (1990)

ZOMPERO, A. F.; LABURU, C. E. As relações entre aprendizagem significativa e representações multimodais. Ensaio, Belo Horizonte (BH), v. 12, n. 03, p. 31-40. 
Recebido: 14 ago. 2019.

Aprovado: 18 ago. 2020.

DOI: $10.3895 /$ rbect.v13n3.10527

Como citar: ASSUNÇÃO, J. A.; MOREIRA, M. A.; SAHELICES, C. C. A resolução de problemas como

estratégia metodológica de ensino, fundamentada na teoria da aprendizagem significativa: uma análise

quantitativa. Revista Brasileira de Ensino de Ciência e Tecnologia, Ponta Grossa, v.13, n. 3, p. 202-223,

set./dez. 2020. Disponível em: <https://periodicos.utfpr.edu.br/rbect/article/view/10527>. Acesso em: XXX

Correspondência: Jeneffer Araújo de Assunção - jenefferassuncao@gmail.com

Direito autoral: Este artigo está licenciado sob os termos da Licença Creative Commons-Atribuição 4.0

Internacional.

\section{(c) (i)}

